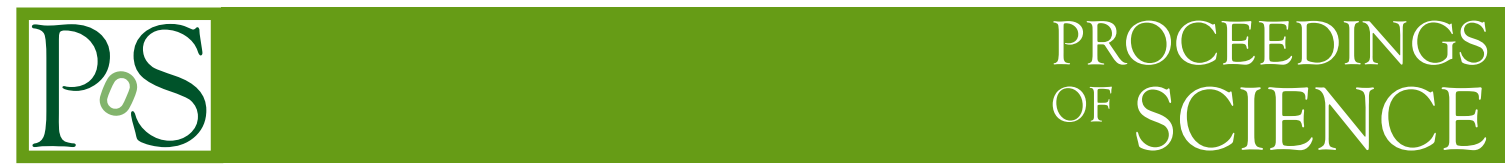

\title{
Plans for experiments protection at the LHC
}

\author{
Massimiliano Ferro-Luzzi* \\ CERN \\ E-mail: massimiliano.ferro-luzzi@cern.ch
}

The Large Hadron Collider (LHC), once in operation, will represent approximately a 200-fold increase in stored beam energy with respect to previous high energy colliders. Safe operation will critically rely on machine and experiment protection systems. We describe the general experiment protection strategy against beam failures with some emphasis on silicon vertex detectors of the LHC experiments.

The 16th International Workshop on Vertex detectors September 23-28, 2007

Lake Placid, NY, USA

${ }^{*}$ Speaker. 


\section{Introduction}

The CERN Large Hadron Collider (LHC [1]) will soon be brought in operation. The proton momentum $(7 \mathrm{TeV} / \mathrm{c})$ and intensity $\left(2808\right.$ bunches $\times 1.15 \times 10^{11}$ protons/bunch) will be such that the total energy stored in one beam, $360 \mathrm{MJ}$, will be more than two orders of magnitude above the maximum beam energy stored in previous high energy colliders, like TEVATRON and HERA. Even during injection into the $\mathrm{LHC}$, at $450 \mathrm{GeV}$, the energy stored in a single nominal batch of protons (288 bunches) will be 2.4 MJ, i.e. in excess of the maximum energy stored in a TEVATRON proton beam $(1.5 \mathrm{MJ})$ or in a HERA proton beam $(2 \mathrm{MJ})$. Equipment damage potential also relates to the energy density of the beam. In this respect, considering the small LHC beam dimensions, the maximum energy density will be about a factor 1000 higher than for other accelerators. To cope with these extreme conditions, a robust machine protection system has been developed for the LHC machine [2].

Past experience with beam accidents in particle physics detectors, particularly in vertex detectors, teach us that experiments should implement a dedicated experiment protection system against beam failures. At the LHC, beyond relying on passive machine protection elements (absorbers and collimators), the experiments will have (i) a stand-alone protection system capable of detecting potentially harmful beam conditions and, when required, triggering a beam abort on the appropriate time scale, (ii) the capability to inhibit injection into the LHC machine, and (iii) the means to monitor particle rates in the experiment during injection and stop the process if necessary.

The purpose of this article is to give an overview of experiment protection at the LHC. Section 2 describes the general LHC layout. Because the machine protection system constitutes the bulk and 'first line of defense' of LHC experiment protection, we outline in section 3 its general strategy and principal features (a more detailed and more expert description can be found in Ref. [1, 2, 3]). Section 4 briefly reviews possible beam failure scenari. In section 5 we describe the main features of the experiment protection systems. The special case of movable detectors is covered in section 6 , while in section 7 we discuss the damage potential of LHC beams. Finally, section 8 gives a summary and outlook.

\section{The LHC machine and experiments}

Figure 1 shows the general layout of the LHC which is divided in eight octants joined by eight insertion regions (IR). Four of these insertion regions (IR1, IR2, IR5 and IR8) are traversing experimental areas. The RF system for beam acceleration is located in IR4. The clockwise beam (beam 1 ) is injected near interaction point 2 (IP2), while the anticlockwise beam (beam 2) is injected near IP8. Apart from a few specific collimators, the collimation system is implemented in IR3 (for momentum cleaning) and IR7 (for betatron cleaning). Beam extraction is implemented in IR6.

Figure 2 shows the layout of two insertion regions. The top figure displays the IR5 layout, similar to IR1, while the bottom figure shows the IR8 layout, similar to IR2. ATLAS [4] and LHCf [5] are installed around IP1, which can be rated the 'safest' of all interacton points in terms of possible beam failure scenari. CMS [6] and TOTEM [7] are located at IP5, one arc away from the beam dump section in IR6. ALICE [8] and LHCb [9] are hosted in IR2 and IR8 which are the regions of beam injection, beam 1 and beam 2 respectively, just about $200 \mathrm{~m}$ away from the 


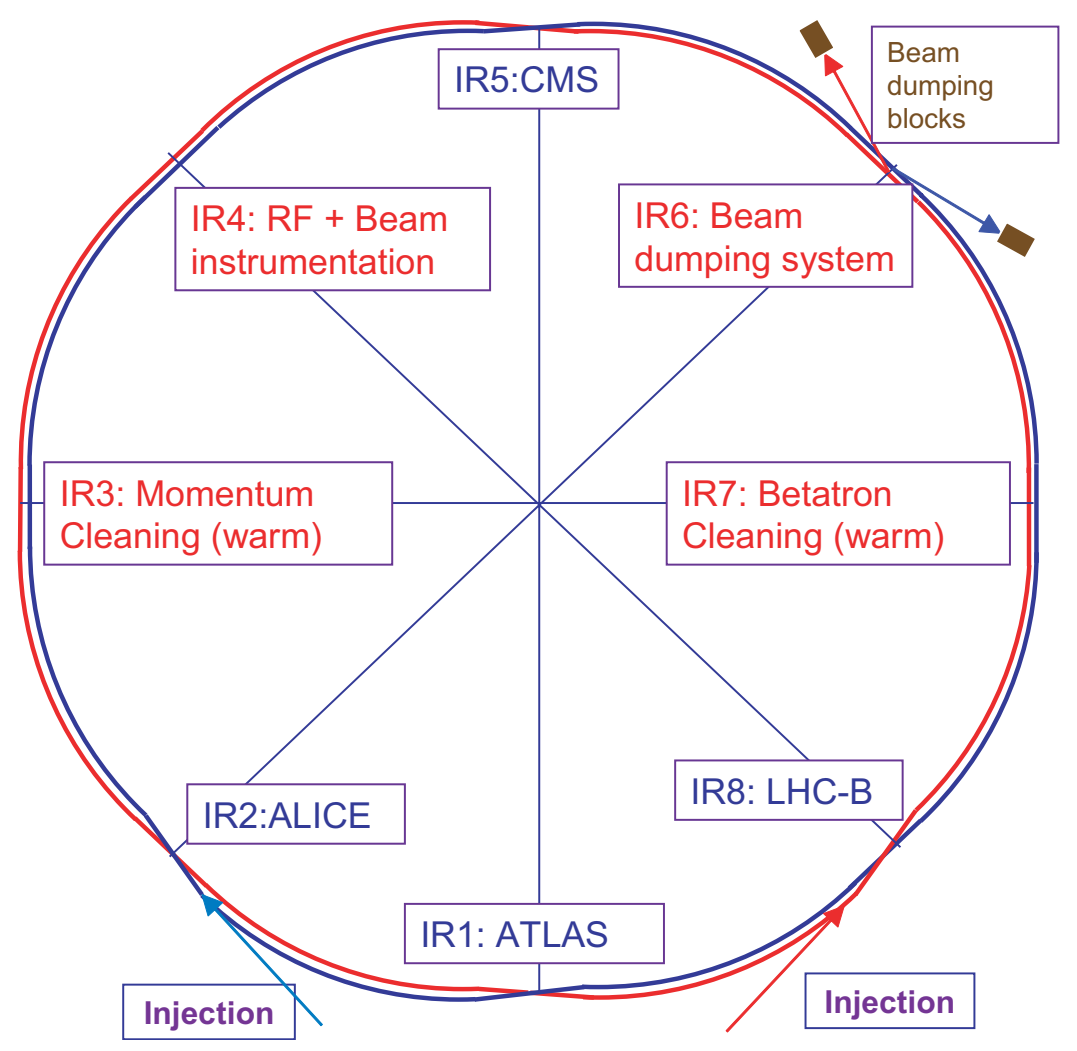

Figure 1: Schematic 'top view' of the LHC (courtesy Rudiger Schmidt, CERN). Beam 1 (clockwise) and beam 2 (anticlockwise) are injected in IR2 and IR8 and both are extracted in IR6. Note that IR1 and IR5 are also hosting forward detector experiments, LHCf [5] and TOTEM [7] respectively (not indicated on the figure).

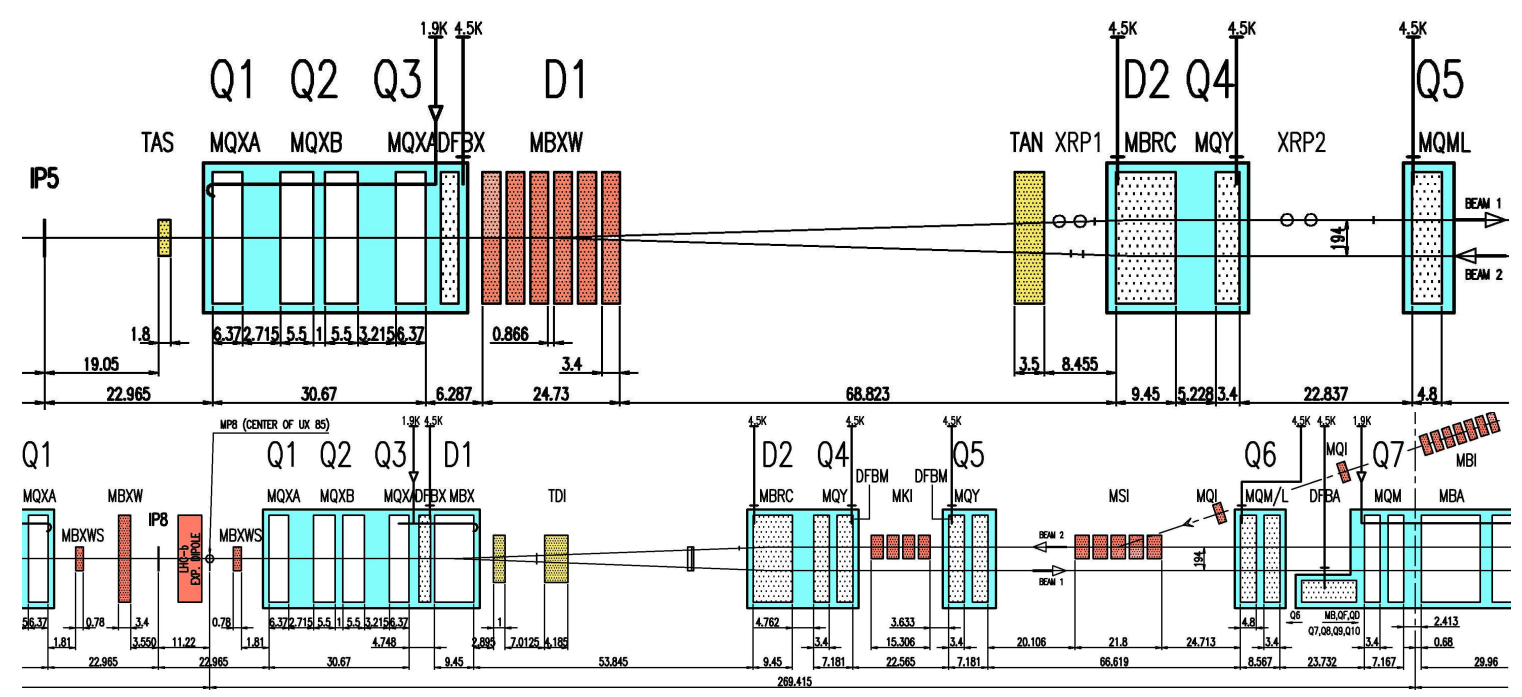

Figure 2: Layout of two insertion regions, IR5 (top) and IR8 (bottom). Warm magnets are indicated in red (MBXW, MBXWS, MKI, MSI, MQI, MBI), cold magnets in light blue (MQXA, MQXB, MBRC, MQY, MQML, MBX, MQM/L, MQM, MBA). Yellow elements indicate absorbers (TAS, TAN) or collimators (TDI). XRP1 \& XRP2 show the positions of the TOTEM Roman Pot stations. Distances are shown in meters. 
IP. Furthermore, ALICE and LHCb each have a dipole spectrometer magnet in the experiment and three compensator magnets deviating the beams in the vertical plane (for ALICE) and ring plane (for LHCb). Finally, ATLAS and CMS have so-called TAS absorbers, which are $1.8 \mathrm{~m}$ long copper blocks situated at $\pm 19 \mathrm{~m}$ from the IP. These are needed to protect the inner triplet of cryogenic quadrupoles around the IP from the excessive heat load due to particles from protonproton collisions. Accessorily, the TAS absorbers also protect the inner detectors of ATLAS and CMS from a variety of beam failures. Due to the lower design luminosity of ALICE and LHCb, the inner triplets in IR2 and IR8 do not require such protection. The different configurations in each experimental area imply that, beyond beam failure scenari common to all, some experiments will be more exposed to specific beam failures.

\section{Machine protection and beam interlock system}

The LHC machine protection system has been described in great details in Ref. [2] and references therein. It relies on both passive and active protection. The former is based on aperture limitation and dilution/absorption of beam losses (by collimators, absorbers, diluters). The latter implements fast detection of problem conditions (beam loss and beam position monitors, quench detectors, etc.) and fast beam extraction (LHC beam dumping system or LBDS).

At the LHC, about $85 \%$ of the $27 \mathrm{~km}$ of the ring circumference is composed of superconducting magnets operated at 1.9 or $4.5 \mathrm{~K}$. The combination of a large stored energy in the beams and a massive usage of cryogenic superconducting magnets requires a sophisticated collimation system with unprecedented performance $[1,10]$. In contrast to other machines such as HERA, RHIC and TEVATRON, the LHC machine cannot be operated without collimation, because of the tight quench margins*. This by itself will ensure a significant level of safety for the experiments: the collimators must define the aperture at all times. For an assumed beam loss lifetime of $10 \mathrm{~h}$, the collimation system must catch with $99.9 \%$ probability the particles that would otherwise be lost on sensitive items, such as the cold aperture (superconducting magnets) or the detectors around the interaction points.

A sketch of the beam interlock system (BIS) is shown in Fig. 3. Two redundant optical loops per beam transport so-called Beampermit signals around the ring. Each pair of loops is composed of a clockwise and anticlockwise propagating signal loop. Two beam interlock controllers (BIC) per insertion region are used to make a logical And of a number of logical signals provided by the users (UserPermit signals). When a UserPermit signal is set False, then the BeamPermit is removed (the optical signal loops interrupted), which fires the dump system and blocks injection from the Super Proton Synchrotron (SPS). For example, beam loss monitors and beam position monitors may detect abnormal conditions and fire a beam dump, or quench protection sensors may detect a developing quench and fire a beam dump. In total, there will be several thousand LHC devices with input to a BIC, which imposes severe availability and reliability levels ${ }^{\dagger}$ [11]. The LHC beam dump system, described in Ref. [1], relies on at least 14 out of 15 kicker magnets firing to extract a beam. The kick amplitude is coupled to an energy tracking system which

${ }^{*}$ The quench levels for slow, continuous losses are expected to be approximately $7 \times 10^{8}$ protons $\mathrm{m}^{-1} \mathrm{~s}^{-1}$ at 450 $\mathrm{GeV}$ and $7.6 \times 10^{6}$ protons $\mathrm{m}^{-1} \mathrm{~s}^{-1}$ at $7 \mathrm{TeV}[1]$.

${ }^{\dagger}$ A fraction of these user inputs may be masked under specific conditions, see section 7 . 


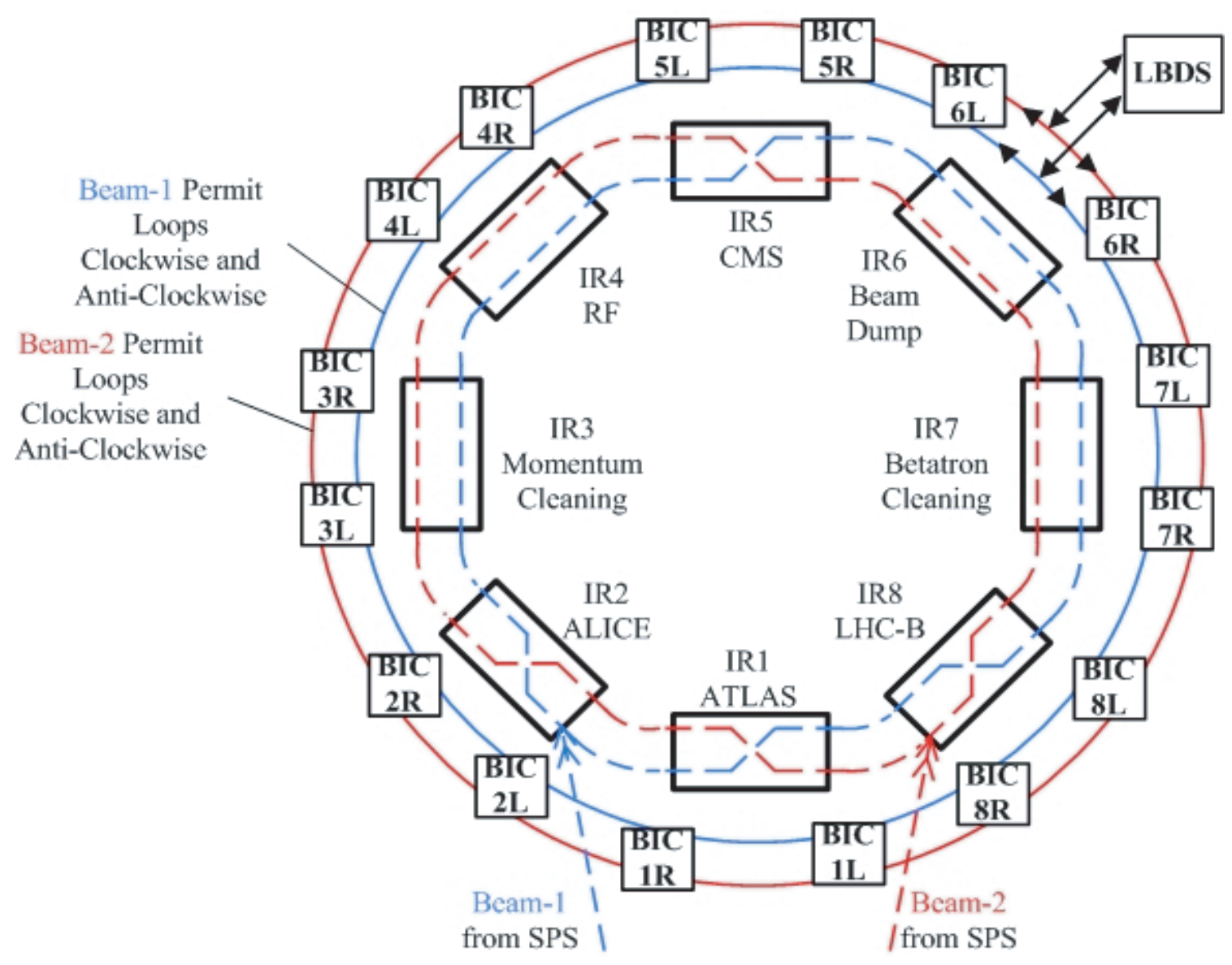

Figure 3: Sketch of the LHC beam interlock system based on optical loops around the ring (extracted from [3], courtesy Rudiger Schmidt, CERN). Explanation in the text.

ensures that beams are properly extracted at any energy [2]. Every beam filling scheme contains an 'abort' gap of at least $3 \mu$ s in the bunch structure, corresponding to the dump kicker rise time. The abort gap in each beam is tracked and monitored. Each beam has an independent dump system. When fired by the BIS, the extraction of the full beam is completed within less than $270 \mu$ s from the removal of the UserPermit signal at the BIC.

\section{Beam failures}

Beam failures can occur on different time scales. Slow, steady losses resulting from beam degradation on the time scale of seconds or minutes may damage the detectors, for instance by increased radiation dose, but do not necessarily require an automated beam abort (recovery of good beam conditions may be attempted). On the contrary, faster losses require rapid, automated reaction via the BIS. For example, beam losses due to a tripping magnet will generally develop on the time scale of several turns ${ }^{\ddagger}$ (for warm magnets) to several milliseconds (for superconducting magnets).

$\ddagger$ One LHC turn is about $89 \mu$ s. 
Ultra-fast losses, on the time scale of one turn or less, are tackled by passive protection. Such losses are due to e.g. an injection failure or a beam extraction failure.

\subsection{Failures with circulating beams}

A large class of beam failure scenari involves circulating beams (at any energy), where beam degradation may be due to a magnet failure or wrong change of settings, to an RF failure, to a collimator failure or wrong change of settings, etc. In these cases beam perturbations will generally affect a large portion of the ring and therefore are likely to be detected by the machine protection system before experiments are affected. An exception to this are possible faults in local bumps, which may affect a single IR with minimal effects outside the local bump region. In this respect, vertex detectors in ALICE and LHCb may be more exposed than those in ATLAS and CMS, as TAS absorbers (for the latter experiments) could help limiting direct hit or high rate splashes to the innermost silicon detectors.

\subsection{Beam failures at injection}

Another class of failures involves beams at injection. Here, an incomplete or unsynchronized kicker fire or wrong magnet settings in the transfer line could detrimentally affect ALICE or LHCb. Wrong magnet settings in the LHC, in particular in any of the experimental IRs (e.g. D1 magnet, see Fig. 2), could cause local damage and affect only the experiment of that particular IR. At 450 $\mathrm{GeV}$, wrong magnet settings can potentially produce much larger deviations on the beam than at top energy. Again, the absence of TAS absorbers and the presence of spectrometer/corrector magnets in ALICE and LHCb may render these two experiments more prone and/or more exposed to such beam failures.

To mitigate the risk for this class of failures, a number of movable and fixed absorbers are placed upstream of the experiments [12]. Furthermore, injection into an empty LHC ring will always start with a single bunch of low intensity, in order to probe at once the settings of all static beam-steering elements of the machine and transfer line and/or to detect unexpected aperture limitations. Once circulating beam is established, injection of high intensity batches may proceed. This procedure is enforced by interlocks [13]. Circulating beam current will be measured in each LHC ring by beam current transformers that will set True an interlock flag (the BeamPresence flags, one per ring $)$ if the measured current is at least $5 \mu \mathrm{A}\left(\approx 2.8 \times 10^{9}\right.$ stored protons $)$. In the SPS injector a similar device sets a flag (SafeLhcInjection flag) which determines whether the prepared beam batch is 'safe' for injection, i.e. whether it is below a certain predefined intensity limit. If this flag is False, then beam transfer from SPS to LHC can only occur if the BeamPresence flag is set True. If SafeLhcInjection is True, beam transfer can occur irrespective of the BeamPresence flag value.

Defining an acceptable value of the safe limit for beam transfer requires a broad understanding of all possible risks involved, for machine equipment as well as for the experiments. The definition of the exact safe beam value for injection is still under discussion, but will probably lie in the range of 5 to $100 \times 10^{9}$ protons. 


\subsection{Beam failures at extraction}

Finally, a class of failures involves beam extraction. A relevant failure of the extraction system for experiments (especially in IR5) is a possible unsynchronized beam abort (kicker pre-fire). In such a case, a number of bunches may be swept during the kicker rise time. Of these bunches, up to 24 may continue their trajectory in the ring, possibly creating large intantaneous losses (mostly caught by collimators). Such beam failures could occur about once per year.

Early simulation studies showed that the IP5 inner triplet and CMS could be severly affected by a kicker pre-fire event [14]. As a consequence of this study, additionnal protective absorbers were added around IR6 in order to largely reduce the impact of such extraction failures $[15,16]$.

\section{LHC experiment protection systems}

The individual LHC experiment protection systems will be centered on diamond-based beam conditions monitors (BCM), a feature common to ALICE, ATLAS, CMS ${ }^{\S}$ and LHCb. Additionnal detectors may participate in the experiment protection system, such as scintillator counters in the case of CMS or Čerenkov counters in ATLAS. The most exposed TOTEM detectors, the Roman Pots, will rely on nearby LHC beam loss monitors for protection [17]. Here, we limit our discussion to the diamond BCM. We outline the general picture of the BCM systems, emphasizing commonality, and refer the reader to the bibliography for the details of each individual BCM $[19,20,18,21]$.

Polycrystalline CVD ${ }^{\mathbb{I l}}$ diamond pads have been selected as the primary sensors of LHC experiment protection for their compactness, simplicity, reliability and radiation resistance. Such sensors have been successfully used at other high energy physics experiments (BABAR [22], BELLE, CDF [23] and ZEUS). BCM diamond pads for LHC experiments were developped first within the RD42 collaboration for ATLAS and CMS and subsequently ported to LHCb and ALICE. For a recent review of diamond detectors in high energy physics applications see Ref. [24].

The LHC experiment beam conditions monitors are generally composed of an array of diamond pads located in the vicinity of the beams, typically 4 or 8 diamond sensors on each side of the IP. Each sensor is about $1 \times 1 \mathrm{~cm}^{2}$ in size and 0.3 to $0.5 \mathrm{~mm}$ thick. Some selected characteristics of the diamond BCM systems of LHC experiments are listed in table 1. Indicatively, the average primary flux of minimum-ionizing particles (MIP) per diamond pad at $r \approx 4 \mathrm{~cm}$ (radius from beam axis) and $z \approx 2 \mathrm{~m}$ (longitudinal distance from IP) are expected to be in the order of 0.05 per inelastic proton-proton interaction, at $14 \mathrm{TeV}$ center of mass energy.

The BCM systems will be operated stand-alone with a dedicated readout chain. The readout schemes and speeds differ from experiment to experiment. As shown in 1, some systems integrate over $\sim 40 \mu \mathrm{s}$, others implement bunch-by-bunch rate capability ( $25 \mathrm{~ns}$ readout speed) which also allows monitoring beam halo by timing. All systems use an FPGA-based readout board to process the data and generate a decision. The detailed algorithms and threshold definitions for actuating a beam dump request are under development. The experiment protection systems will dump both beams when generating a beam abort.

\footnotetext{
$\S$ The TOTEM trackers T1 and T2, are mechanically integrated in CMS and protected by the CMS BCM [17].

${ }^{\mathrm{I}}$ Chemical vapor deposition.
} 


\begin{tabular}{llcccccc}
\hline \hline \multicolumn{2}{c}{ System } & $\begin{array}{c}\text { Pads } \\
\text { per IP side }\end{array}$ & $\begin{array}{c}\text { Radial distance } \\
\text { from beam line }\end{array}$ & $\begin{array}{c}\text { Longitudinal } \\
\text { distances from IP }\end{array}$ & Readout & Ref. \\
\hline \multirow{2}{*}{ ALICE } & BCM & 8 & $5.8 \mathrm{~cm}$ & $-15.6 \mathrm{~m}$ & - & $40 \mu \mathrm{s}$ & [18] \\
& & 8 & $6.8 \mathrm{~cm}$ & - & $+19.08 \mathrm{~m}$ & $40 \mu \mathrm{s}$ & \\
\multirow{2}{*}{ ATLAS } & BCM1 & 4 & $7 \mathrm{~cm}$ & $-1.83 \mathrm{~m}$ & $+1.83 \mathrm{~m}$ & $25 \mathrm{~ns}$ & [19] \\
& BCM2 & 6 & $6.5 \mathrm{~cm}$ & $-3.43 \mathrm{~m}$ & $+3.43 \mathrm{~m}$ & $40 \mu \mathrm{s}$ & \\
\multirow{2}{*}{ CMS } & BCM1 & 4 & $4.3 \mathrm{~cm}$ & $-1.85 \mathrm{~m}$ & $+1.85 \mathrm{~m}$ & 5 to $10 \mu \mathrm{s}$ & [20] \\
& BCM2 & 12 & 5 and $29 \mathrm{~cm}$ & $-14.4 \mathrm{~m}$ & $+14.4 \mathrm{~m}$ & $40 \mu \mathrm{s}$ & \\
\multirow{2}{*}{ LHCb } & BCM-U & 8 & $\sim 3 \mathrm{~cm}$ & $\sim-2 \mathrm{~m}$ & - & $40 \mu \mathrm{s}$ & {$[21]$} \\
& BCM-D & 8 & $3.1 \mathrm{~cm}$ & - & $+2.8 \mathrm{~m}$ & $40 \mu \mathrm{s}$ & \\
\hline \hline
\end{tabular}

Table 1: Selected characteristics of CVD diamond beam conditions monitors of LHC experiments. (Note that, to avoid confusion with LHC beam loss monitors, we have changed here the official ATLAS names 'BCM' and 'BLM' to BCM1 and BCM2.)

Given the fact that all experiment will use a non-maskable input to the local BIC, the LHC machine will not operate if any of the experiment UserPermit signals is missing. This imposes strong availability requirements on the experiment protection system, in particular the BCM, which is required to be ready from the first day of LHC operation. All BCM systems are powered by uninterruptable power supply. Interestingly, the CMS BCM system will be powered from an electrical switchboard belonging to the LHC machine, thus ensuring that the BCM availability is matched with beam availability rather than with the experiment up-time. Other experiments are now considering this possibility. The experiment protection systems are required to implement 'post-mortem' data retrieval and analysis that allows reconstructing a posteriori the few milliseconds preceding any beam abort.

In general, the primary purpose of the BCM systems is to protect the experiments against circulating beam failures. Nonetheless, the experiments are considering the use of BCM systems and others detectors to monitor possible abnormal rates at all times, in particular during injection, to generate a feedback warning for the experiment and LHC control rooms and/or to inhibit further injection if necessary.

\section{The special case of movable detectors}

Several experiments will make use of movable detectors in the LHC machine. These require special interlock functionality in order to reduce the risk of beam damage when the detectors are in the closed position for physics. TOTEM will use silicon strip detectors in pairs of Roman Pot devices located at $z \approx \pm 147 \mathrm{~m}$ and $z \approx \pm 220 \mathrm{~m}$ from IP5 [7]. These Roman Pots consist of movable vacuum enclosures that enable bringing the silicon sensors to a distance of $1.2 \mathrm{~mm}$ from the beam. ATLAS will implement scintillating fiber detectors in a similar configuration in IR1 [25], though not before the year 2009. The LHCb vertex locator (VELO [26]) at IP8, composed of 42 silicon strip modules mounted in the vacuum, is also a movable detector. It is divided in two halves (left 
and right of the beams) that can be retracted sideways by $30 \mathrm{~mm}$ during LHC filling. The 21 VELO modules of each half are enclosed in a thin-walled Aluminium box $(250 \mu \mathrm{m})$ that separates the beam vacuum from the detector vacuum. In nominal physics operation the silicon detectors will be precisely positioned around the colliding beams and the box material will approach the beams to a mere $5 \mathrm{~mm}$ distance (the silicon edges reaching a radial distance of about $7 \mathrm{~mm}$ ).

Because of the expected beam excursions during beam filling and preparation for physics, all movable detectors are required to be in the retracted (or 'garage') position during these operations. Beam modes have been defined to characterize the operational states. Interlock flags derived from the beam modes will be transmitted to the experiment protection systems for conditioning their interlocks. One particular flag will signal when movable detectors are allowed to leave their garage position. If this flag is set False and a movable detector is not in garage position, then the experiment protection system will fire a beam dump. Furthermore, whenever a movable detector is not in garage position the corresponding experiment will inhibit injection from the SPS.

Protection of movable detectors critically relies on the experiment or machine protection systems (BCM or BLM). A local excursion of a circulating beam, or a failure in the motion system of a movable detector, may dangerously bring the beam envelope in overlap with the detector enclosure or other nearby elements. The motion systems are too slow to react on such eventualities. Therefore, these abnormal conditions must be detected by the BCM (or BLM) and instantly lead to a beam dump trigger.

\section{Damage potential of LHC beam failures}

All vertex detectors and most inner trackers at LHC experiments are based on silicon technology. Beam-induced damage for silicon detectors may have different causes, among which: heat deposition, radiation damage and charge-induced breakdown.

- Heat deposition: A crude estimatell suggests that an instantaneous rate of $R \approx 10^{13}$ protons $/ \mathrm{cm}^{2}$ will not increase the local temperature of silicon by more than a few degrees (neglecting particle showering in the silicon). Given the lightness of silicon vertex detectors and the assumed beam failure scenari, heat-induced damage to silicon seems unlikely.

- Radiation damage: Incurred displacement damage will 'eat up' the radiation dose budget. However, silicon detectors at LHC experiments are designed to sustain fluences of up to several $10^{14} n_{\mathrm{eq}} / \mathrm{cm}^{2 * *}$, corresponding to an absorbed dose of about 10 Mrad. Example studies by ALICE [27] and ATLAS [28] indicate that, given the assumed scenari and occurence probabilities, increased radiation dose due to beam failures is not expected to significantly cut down the detector life time. Nonetheless, all experiments will carry out detailed monitoring of radiation fluences, so that minimization of radiation damage may be attempted by beam tuning.

- Charge-induced breakdown: Sudden high rate may drastically change the electric field configuration in silicon, which locally becomes conductive, and possibly destroy local features

${ }_{\Delta T} \approx\left(1.66 \mathrm{MeV} \mathrm{cm}^{2} / \mathrm{g}\right) \cdot R / C_{p}=3.8 \mathrm{~K}$, with a specific heat of silicon $C_{p} \approx 0.7 \mathrm{Jg}^{-1} \mathrm{~K}^{-1}$.

${ }^{* *}$ Equivalent non-ionizing energy loss: $1 n_{\mathrm{eq}}=1 \mathrm{MeV}$ neutron equivalent displacement damage in silicon. 
of the sensors, depending on the technology used. For example, the bias voltage may be moved to across a silicon oxide dieletric layer between strip implant and readout strip. The silicon oxide layer breaks down at about $1 \mathrm{~V} / \mathrm{nm}$ and thus, depending on the particle rate, the sensor may be locally damaged (e.g. production of pin holes). A direct hit to front-end integrated circuits may cause even greater damage, as the loss of a readout chip generally implies the loss of many detector channels.

The damage potential of an LHC beam for bulk inactive material has been studied by simulations and cross-calibrated with a controlled experiment at injection energy [29]. SPS batches of increasing intensity were directed into a stack target of selected materials. The result of these studies were found in reasonable agreement with simulations (at the $30 \%$ level) and indicated that, for copper, the melting point was reached at about $2.4 \times 10^{12}$ protons and clear damage became visible at about $4.8 \times 10^{12}$ protons. These studies led to a definition of the SafeBeam value for LHC equipment at injection energy ( $10^{12}$ protons) and, based on simulation, at top energy $\left(10^{10}\right.$ protons). Below this value, the number of active inputs to the BIS may be relaxed by masking specific inputs.

Concerning silicon detectors, high particle rate tests were performed at the CERN Proton Synchrotron (PS) with batches of $24 \mathrm{GeV}$ bunches by ATLAS [30] and CMS [31]. Proton bunches ${ }^{\dagger \dagger}$ were directed onto silicon detector modules, with peak bunch densities in the order of $3 \times 10^{10}$ protons $/ \mathrm{cm}^{2}$. The detectors were under bias and the front-end electronics were kept under voltage. Both groups concluded that LHC beam losses producing particle rates of up to $3 \times 10^{10}$ protons $/ \mathrm{cm}^{2}$ in about $40 \mathrm{~ns}$ would not cause irreversible damage to the studied silicon detectors, although a reset of the front-end electronics may be required. Furthermore, laboratory tests were carried out to infer from the response to laser beam pulses the damage potential of high MIP rates on ATLAS silicon strip detectors under bias [32]. Damage to aluminium readout strips was observed at laser pulse densities corresponding to rates of the order of $10^{9}$ MIP in $6 \mathrm{~ns}$ injected in a single strip.

Quite generally, detector components in the experiments, in particular close to the beam line, such as silicon sensors, may not be as sturdy as machine equipment. Although the actual damage limits (in terms of MIP $\mathrm{cm}^{-2} \mathrm{~ns}^{-1}$ ) of silicon detectors used in LHC experiments is yet unclear, recent experience with TEVATRON or LEP experiments [33] would suggest reducing the limit for SafeLhcInjection to the 'lowest possible value'. However, LHC beam instrumentation is limited in sensitivity, which precludes efficient machine studies at intensities below about $3 \times$ $10^{9}$ protons. In addition, dealing with bunches of such small intensity may require time costly adjustements in the injector chain. Therefore, a trade-off value for the SafeLhcInjection flag must be found, which soundly balances experiment protection and machine operation efficiency.

\section{Summary and outlook}

In summary, with the Large Hadron Collider a new domain for stored beam energy is entered which imposes extreme requirements on machine and experiment protection. The installation of these protection systems is currently being completed and commissioning has started on some subsystems.

\footnotetext{
${ }^{\dagger}$ Bunch length $42 \mathrm{~ns}$, bunch intensity $\approx 10^{11}$ protons and bunch separation of $256 \mathrm{~ns}$.
} 
The damage risk for silicon vertex detectors depends on the detailed design of the sensors (pixels versus strips, $p$-in- $n$ versus $n$-in- $n$, AC versus DC coupling, geometry, etc.) which broadly varies across LHC experiments. It may as well depend on the state of the detector (value of silicon bias voltage, state of front-end chip supply voltage, etc.). A detailed characterization of the most exposed detectors in each experiment and good understanding of the risks associated with possible beam failures can lead to a better policy of operation of these detectors when the LHC is not in stable beam conditions. For instance, the advantages of detector stability (no charge up effects, no temperature excursions, etc., when all voltages are kept on at all times) will have to be weighed against a possible risk increase for the detectors in situations where beams are not ready for physics.

Further detector tests and beam failure simulation studies will help refining the operation policy of the machine and detectors, and defining initial dump thresholds, especially during the beam commissioning phase.

\section{Acknowledgments}

The author would like to stress that the presentation herein covers the work of a large number of people. Presumably, he has not been able to do justice on all the excellent work done on the development and construction of the LHC machine and experiment protection systems, and apologises for the inevitable ommissions. Furthermore, he would like to address special thanks to Mario Deile, Antonello Di Mauro, Richard Jacobsson, Daniela Macina, Alick Macpherson, Siegfried Wenig and Jörg Wenninger for their help in preparing the presentation for the Vertex 2007 Workshop and for reading this manuscript before publication.

\section{References}

[1] O. Brüning, P. Collier, P. Lebrun, S. Myers, R. Ostojic, J. Poole and P. Proudlock editors (Geneva, CERN, 2004), LHC Design Report v.1: the LHC Main Ring, CERN-2004-003-V-1a.

[2] R. Schmidt et al., Protection of the CERN Large Hadron Collider, New J. of Phys. 8 (2006) 290.

[3] B. Todd, A Beam Interlock System for CERN High Energy Accelerators, Ph.D. thesis, Brunel University, West London, and CERN-THESIS-2007-019, Geneva, Switzerland, October 2006.

[4] W. W. Armstrong et al.(The ATLAS Collaboration), ATLAS: Technical Proposal for a GeneralPurpose pp Experiment at the Large Hadron Collider at CERN, CERN-LHCC-94-43 (1994), http://cdsweb.cern.ch/record/290968.

[5] O. Adriani et al.(The LHCf Collaboration), LHCf Experiment: Technical Design Report, CERN-LHCC-2006-004 (2006), http://cdsweb.cern.ch/record/926196.

[6] M. Della Negra, L. Foà, A. Hervé, A. Petrilli et al.(The CMS Collaboration), CMS Physics: Technical Design Report. Vol. 1: Detector Performance and Software, CERN-LHCC-2006-001 (2006), http://cdsweb.cern.ch/record/922757.

[7] V. Berardi et al.(The TOTEM Collaboration), Total Cross-Section, Elastic Scattering and Diffractive Dissociation at the Large Hadron Collider at CERN: TOTEM Technical Design Report, CERN-LHCC-2004-002 (2004), http://cdsweb.cern.ch/record/704349. 
[8] F. Carminati et al.(The ALICE collaboration), ALICE: physics performance report, volume I, J. Phys. G 30 (2004) 1517; B. Alessandro et al.(The ALICE collaboration), ALICE: physics performance report, volume II, J. Phys. G 32 (2006) 1295.

[9] S. Amato et al.(The LHCb Collaboration), LHCb Technical Proposal: a Large Hadron Collider Beauty Experiment for Precision Measurements of CP Violation and Rare Decays, CERN-LHCC-98-004 (1998), http://cdsweb.cern.ch/record/622031.

[10] R. Assmann et al., The Final Collimation System for the LHC, 10th European Particle Accelerator Conf. EPAC 2006 (Edinburgh, 26-30 June 2006), CERN-LHC-PROJECT-Report-919 .

[11] R. Filippini et al., Reliability Assessment of the LHC Machine Protection System, in Proc. of the 2005 Particle Accelerator Conference, Knoxville, Tennessee, p. 1257; R. Filippini et al., Reliability Analysis of the LHC Beam Dumping System, in Proc. of the 2005 Particle Accelerator Conference, Knoxville, Tennessee, p. 1201; A.V. Fernández, F. Rodríguez-Mateos, Reliability of the quench protection system for the LHC superconducting elements Nucl. Instrum. Methods Phys. Res. A 525 (2004) 439.

[12] V. Kain, What is required to safely fill the LHC, in 3rd LHC Project Workshop, Divonne-les-Bains, France, Jan 2006, CERN-AB-2006-014, J. Poole editor, http://cdsweb.cern.ch/record/939516.

[13] B. Puccio, R. Schmidt, J. Wenninger, Beam Interlocking Strategy between the LHC and its Injector, in Proc. of the 10th ICALEPCS Int. Conf. on Accelerator \& Large Expt. Physics Control Systems., Geneva, 10 - 14 Oct 2005, http://epaper.kek.jp/ica05/INDEX.HTM.

[14] A.I. Drozhdin, N.V. Mokhov, M. Huhtinen, Impact of the LHC beam abort kicker prefire on high luminosity insertion and CMS detector performance in Proc. of the 18th Biennial Particle Accelerator Conference, New York (1999), p. 1231.

[15] B. Goddard, R.W. Assmann, E. Carlier, J. Uythoven, J. Wenninger, W.Weterings, Protection of the LHC against Unsynchronised Beam Aborts, in Proc. of the European Particle Accelerator Conference, Edinburgh, Scotland, UK, 26 - 30 Jun 2006 (LHC-PROJECT-Report-916);

[16] Report on final simulation results for the impact of LHC extraction kicker pre-fire is in preparation (B. Goddard, private communication).

[17] M. Deile, private communication.

[18] A. Di Mauro, private communication, and H. Schindler, Protecting the ALICE experiment from beam failures, Master Thesis, Technische Universität, Wien, to be published.

[19] S. Wenig, private communication; M. Mikuž et al., Diamond Pad detecor telescope for beam conditions and luminosity monitoring in ATLAS, Nucl. Instrum. Methods Phys. Res. A 579 (2007) 788; A. Gorišek, et al., ATLAS diamond Beam Condition Monitor, Nucl. Instrum. Methods Phys. Res. A 572 (2007) 67; H. Pernegger, H. Frais-Kölbl, E. Griesmayer and H. Kagan, Design and test of a high-speed single-particle beam monitor, Nucl. Instrum. Methods Phys. Res. A 535 (2004) 108.

[20] A. Macpherson, private communication; L. Fernandez-Hernando et al., Development of a CVD diamond Beam Condition Monitor for CMS at the Large Hadron Collider, Nucl. Instrum. Methods Phys. Res. A 552 (2005) 183.

[21] C.J. Ilgner, private communication, and C.J. Ilgner Implementation of a Diamond-Beam-Conditions Monitor into the LHCb Experiment at CERN, Nuclear Science Symposium Conference Record, 2007, IEEE, p. 1700.

[22] A.J. Edwards et al., Radiation monitoring with CVD diamonds in BABAR, Nucl. Instrum. Methods Phys. Res. A 552 (2005) 176. 
[23] R. Eusebi et al., A Diamond-Based Beam Condition Monitor for the CDF Experiment, Nuclear Science Symposium Conference Record, 2006, IEEE Vol. 2, p. 709.

[24] R.S. Wallny, Status of diamond detectors and their high energy physics application, Nucl. Instrum. Methods Phys. Res. A 582 (2007) 824.

[25] See e.g. B.D. Girolamo, Luminosity measurement at ATLAS with Roman Pots and scintillating fibre detectors, Nucl. Instrum. Methods Phys. Res. A 581 (2007) 526.

[26] See e.g. M.G. van Beuzekom, The LHCb Vertex Locator: Present and future, Nucl. Instrum. Methods Phys. Res. A 579 (2007) 742.

[27] P. Giubellino, A. Morsch, L. Leistam, B. Pastirčák, Radiation in ALICE from a misinjected beam to LHC, CERN-ALICE-INT-2001-03.

[28] D. Bocian, Accidental Beam Losses during Injection in the Interaction Region IR1, CERN-LHC-Project-Note-335.

[29] V. Kain, K. Vorderwinkler, J. Ramillon, R. Schmidt, J. Wenninger, Material damage test with $450 \mathrm{GeV}$ LHC-type beam, in Proc. of the 2005 Particle Accelerator Conference, Knoxville, Tennessee, p.1607.

[30] A. Andreazza, K. Einsweiler, C. Gemme, L. Rossi, P. Sicho, Effect of accidental beam losses on the ATLAS pixel detector, Nucl. Instrum. Methods Phys. Res. A 565 (2006) 50.

[31] M. Fahrer, G. Dirkes, F. Hartmann, S. Heier, A. Macpherson, Th. MÃijller, Th. Weiler, Beam-loss-induced electrical stress test on CMS Silicon Strip Modules, Nucl. Instrum. Methods Phys. Res. A 518 (2004) 328.

[32] K. Hara, T. Kuwano, G. Moorhead, Y. Ikegami, T. Kohriki, S. Terada and Y. Unno, Beam splash effects on ATLAS silicon microstrip detectors evaluated using 1-w Nd: YAG laser, Nucl. Instrum. Methods Phys. Res. A 541 (2005) 15.

[33] For CDF, see e.g. J. Spalding, TEVATRON commissioning and interaction with experiments, in third meeting of the TEV4LHC Workshop, CERN, April 28-30 2005,

http://mlm.home.cern.ch/mlm/TEV4LHC.html and http://indico.cern.ch/conferenceDisplay.py?confId=a052004;

for LEP, see e.g. J. Rothberg, Limitations due to Backgrounds at LEP1, in Proc. of the 5th Workshop on LEP Performance, Chamonix, January 1995, CERN-SL-95-08-DI CERN SL/95-08 (DI), J. Poole editor, http://cdsweb.cern.ch/record/277821, or also in Chapter XIII / section 5 of The ALEPH handbook: 1995, vol. 2, C. Bowdery editor. 